\title{
Double-Fold Operation with a Partial Incision and Continuous Buried Suture
}

\author{
In Sook Kang ${ }^{1}$, Ji Seon Choi ${ }^{1}$, \\ Jeong-geun Hong ${ }^{2}$ \\ ${ }^{1}$ Department of Plastic and \\ Reconstructive Surgery, St. Vincent's \\ Hospital, College of Medicine, The \\ Catholic University of Korea, Seoul; \\ ${ }^{2}$ Metro Plastic Surgery Clinic, Incheon, \\ Korea
}

No potential conflict of interest relevant to this article was reported.

\begin{abstract}
Background The double fold operation is one of the most common aesthetic surgeries in Korea. There are many methods to make double fold using non-incision, incision and partial incision. Patients seeking double folds prefer natural appearance, less downtime, minimal scarring technique. The most favorable method is the minimal invasive but long-lasting fold formation technique.

Methods From October 2003 to September 2016, this procedure was applied in 7,963 patients who worried about surgical scar or preferred non-incision method with puffy eyelids. Five stab incisions including two small incisions were taken in the upper eyelid. Through the small incision lines, the pretarsal muscle and the orbital fat were removed to facilitate tissue adhesion. The double fold line was made with the continuous buried suture. The suture was tied in the fifth stab incision site and the knot was placed deeply to prevent granuloma formation.

Results This method was performed in all patients. The complications were asymmetry (52 cases), fold loosening ( 43 cases), granuloma (12 cases), conjunctival hemorrhage (5 cases), and scar (4 cases). Especially $74 \%$ of fold loosening cases were developed in mild ptotic patients.

Conclusions This method achieves good cosmetic result, shorter operation time, less morbidity, faster recovery in puffy eyelids than the other method.
\end{abstract}

Keywords Blepharoplasty, Double fold, Eyelids, Suture techniques

\section{INTRODUCTION}

The double-fold operation is one of the most popular cosmetic surgical procedures in Korea. Various techniques have been developed to overcome the shortcomings of existing methods and to meet the needs of patients [1]. Although the criteria for beautiful eyes have gradually changed over time, in recent years, those who wish to undergo this procedure generally desire a natural-looking double eyelid shape, rather than a strong fold, and prefer to undergo a technique involving minimal scarring and a short healing

Received: Sep 14, 2017 Revised: Sep 27, 2017 Accepted: Sep 27, 2017 Correspondence: Jeong-geun Hong Metro Plastic Surgery Clinic, 6F, 16 Gwangjang-ro, Bupyeong-gu, Incheon 21404, Korea.

E-mail: joadr@nate.com

Copyright @ 2017 The Korean Society for Aesthetic Plastic Surgery.

This is an Open Access article distributed under the terms of the Creative Commons Attribution Non-Commercial License (http://creativecommons.org/licenses/by-nc/4.0/) which permits unrestricted non-commercial use, distribution, and reproduction in any medium, provided the original work is properly cited. www.e-aaps.org time. Therefore, the non-incision technique is widely practiced because it is relatively simple, causes less scarring than the incision technique, provides a quick recovery, and allows an easy correction. However, in comparison with the incision technique, folds are more likely to disappear, it is difficult to apply to puffy eyelids with excessive soft tissue, and there is a high incidence of inclusion cysts and foreign body granuloma due to knots [2].

A partial incision technique is the best way to obtain a permanent fold through a minimally invasive procedure, and various partial incision techniques have been published, combining the advantages of both the incision and non-incision methods [2].

Partial incision techniques have made it possible to create a strong pretarsal adhesion by debulking the pretarsal soft tissue and creating a long-lasting fold, which is a simple procedure, in contrast to the full-incision method [3].

In this study, the authors developed a standardized method combining the partial incision technique and the suture method, leading to satisfactory results. Thus, we report this technique and provide a literature review. 


\section{METHODS}

From October 2003 to September 2016, this procedure was applied to 7,963 patients who were concerned about surgical scarring or preferred the non-incision method despite having puffy eyelids. In this method, the fold height was designed according to the surgeon's judgment and the patient's preferences. After the infiltration of a local anesthetic, 5 stab incisions including 2 small $(4-5 \mathrm{~mm}$ ) incisions were made along the designed line. Two small incisions were made in the upper eyelid just above the medial and lateral limbus to avoid injuring the levator aponeurosis and to obtain symmetry conveniently. Of particular note, the lateral limbus incision made it possible to identify the aponeurosis, which helped reduce the likelihood of injury. Through the incision site, the pretarsal orbicularis oculi muscle and the orbital fat were removed to facilitate tissue adhesion when indicated. Then, a 7-0 nylon continuous suture was inserted through the 5 incision sites, entering and exiting the conjunctiva. After passing through the conjunctiva, the same needle was passed back through the full layer of the lid, entering through the next hole on the conjunctival surface and exiting via the stab incision previously made on the skin. A continuous suture was performed in the same manner, and tied at the fifth stab incision site. The knot was placed deeply to prevent granuloma formation (Fig. 1).

\section{RESULTS}

Over the course of 13 years, 7,963 patients underwent this doublefold operation using the partial incision and continuous buried suture technique. The average operative time was 20 minutes. This technique showed excellent results in terms of inconspicuous scarring and edema during a 1 to 8-year follow-up period (Fig. 2-5). Four patients had a scar on the incision line due to electrocautery for fat removal, and 52 patients showed various degrees of asymmetry. Twelve patients complained about granuloma formation or suture material exposure, and these complications were corrected easily and satisfactorily. Forty-three patients showed fold loosening, and all required an additional operation. Thirty-two of the 43 patients who experienced fold loosening had mild ptosis preoperatively. Five conjunctival hemorrhages disappeared spontaneously within 2 to 3 weeks.

\section{DISCUSSION}

Half of Asians do not have a double eyelid, due to anatomical structures such as the presence of thick subcutaneous fat tissue in the upper eyelid and a lack of cutaneous insertion of the levator muscle [4]. The double-fold operation is performed to create a definite adhesion in eyelids lacking adhesion between the skin, the levator aponeurosis, and the tarsus, resulting in a double eyelid. Since Mikamo developed the double eyelid blepharoplasty technique in Asians in 1896 [5], the double-fold operation has been further refined, and options now include the incision, non-incision, and partial incision techniques [6].

The incision technique is a method in which a full skin incision is made, the excess muscle is dissected and removed, along with the orbital fat if necessary, and the fold is created through an appropriate fixation between the dermis, levator muscle, and tarsus according to the preferences of the surgeon and patient. This method has the advantage of enabling control of the skin, subcutaneous fat tis-

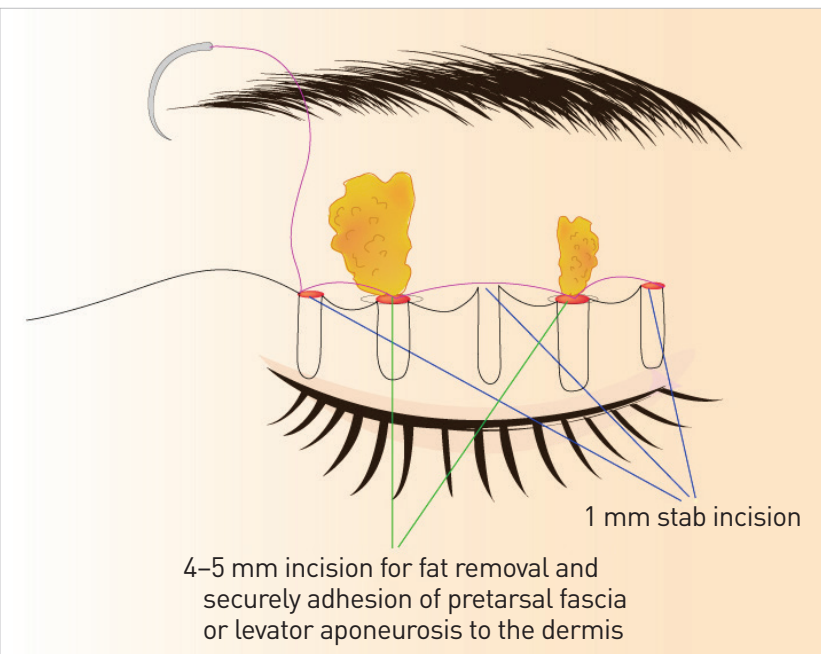

(A)

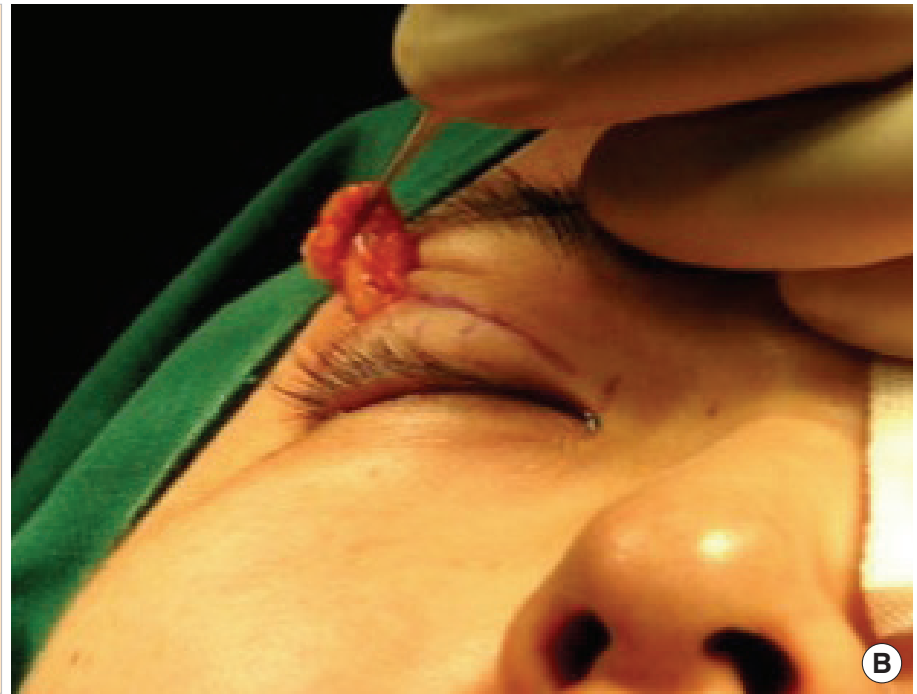

Fig. 1. Continuous buried suture method with a partial incision. (A) A schematic diagram of the procedure. (B) Removing fat through the small lateral incision. 


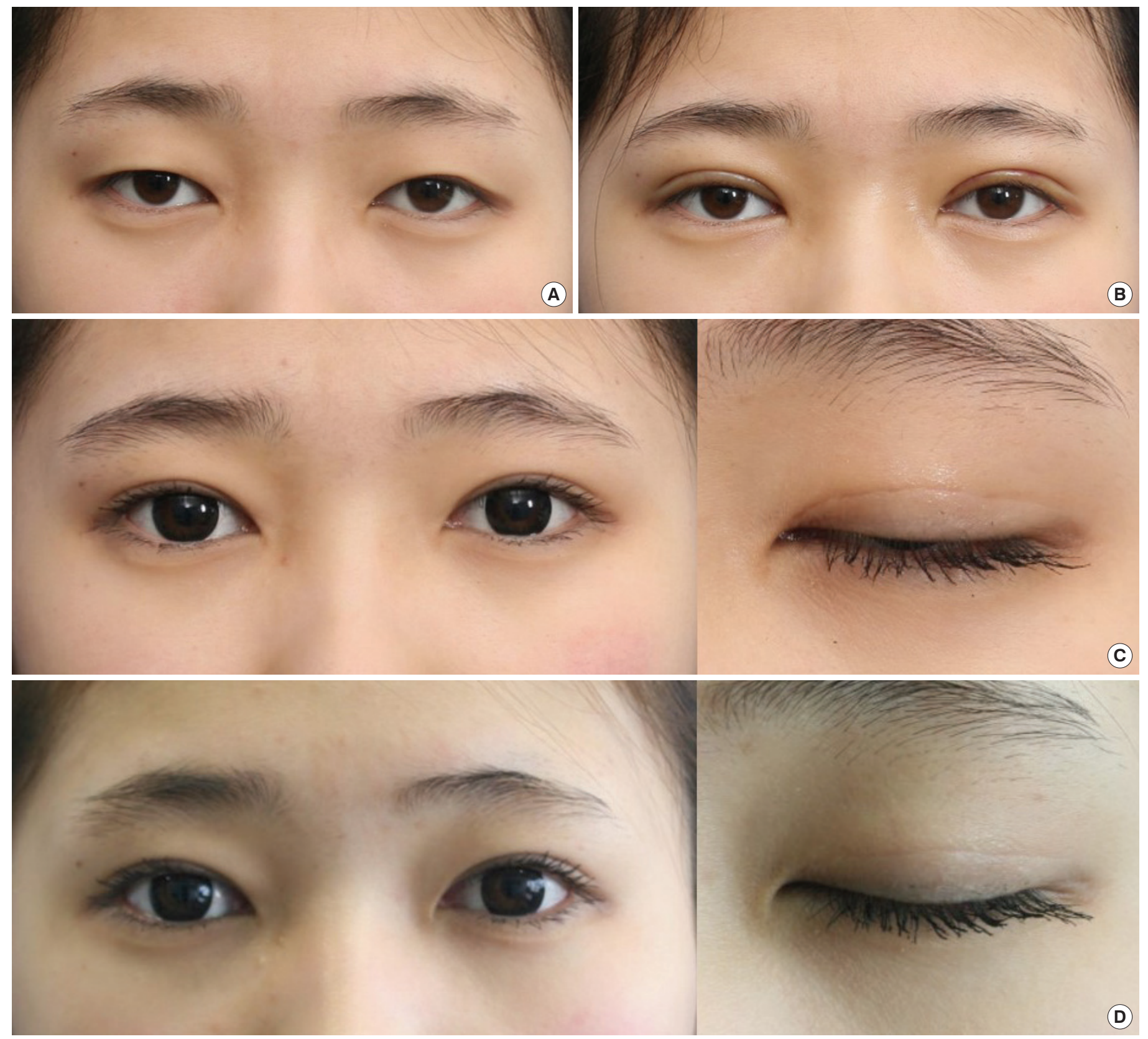

Fig. 2. (A) Preoperative appearance. (B) Appearance 2 days postoperatively. (C) Appearance 1 month postoperatively. (D) Appearance 3 months postoperatively.
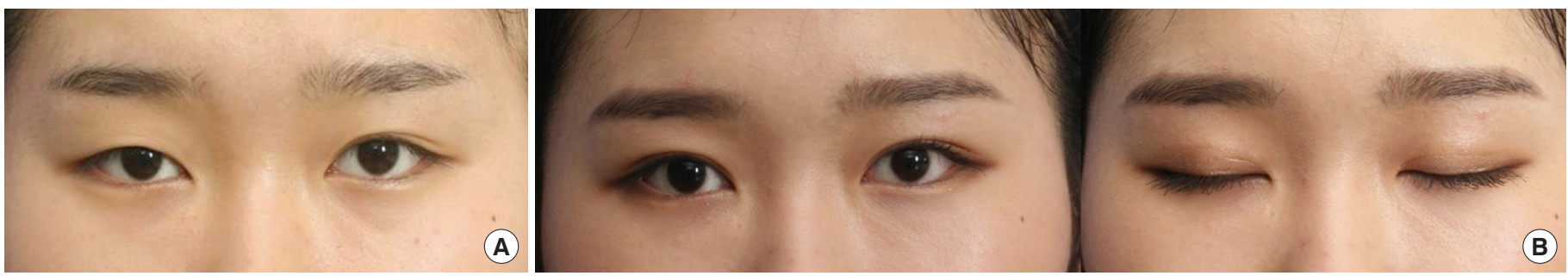

Fig. 3. (A) Preoperative appearance. (B) Appearance 3 months postoperatively. 

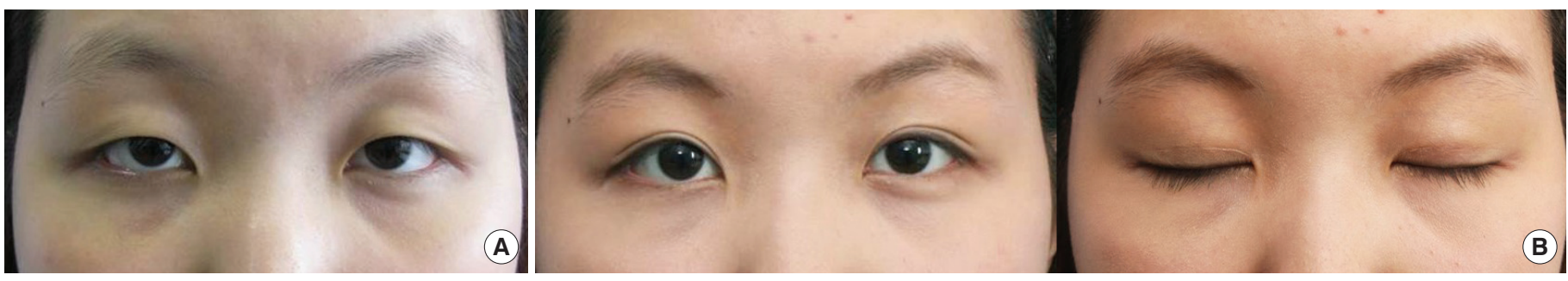

Fig. 4. (A) Preoperative appearance. (B) Appearance 3 months postoperatively.
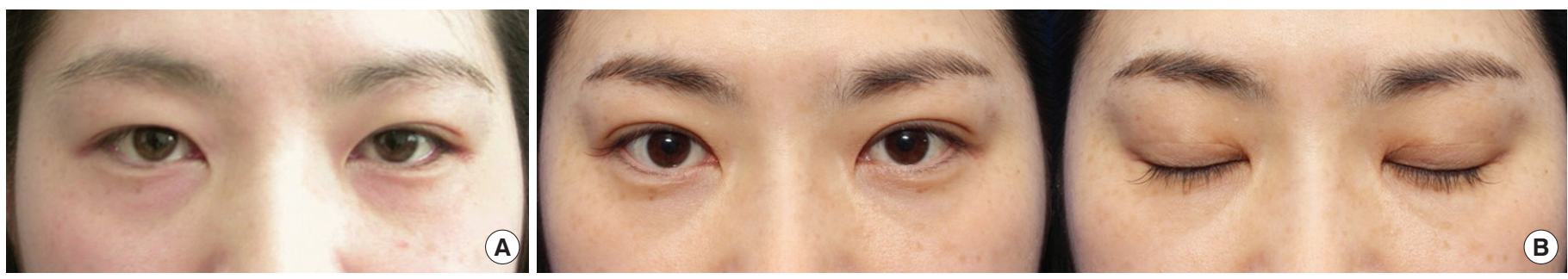

Fig. 5. (A) Preoperative appearance. (B) Appearance 8 years postoperatively.

sue, and levator muscle, and of making a more durable fold, but it has the disadvantage of creating a long scar and requiring a long recovery time [7]. The non-incision technique allows a single continuous suture or several sequential sutures to pass through several skin stab incisions and the conjunctiva without the removal of soft tissue to create a fixation between the tarsus and deep dermis, resulting in fold formation. The suture knots are buried, and a skin suture is usually not needed. This method has the advantages of quick recovery and less scarring, but it has the disadvantages that controlling each element is difficult and there is a strong possibility of fold loosening. Moreover, there are concerns about inclusion cyst or granuloma formation due to the knots [8].

The partial incision technique is a method that takes advantage of both techniques. The small incision technique [3,9,10], 2-minute incision technique [8], and 3-mini-incision technique $[2,11]$ have been introduced. The basic principle is that it is possible to make a stronger pretarsal adhesion than in the non-incision method and to perform more accurate fixation in a more secured view by removal of the orbital fat or pretarsal orbicularis muscle through a small incision, although there are slight differences among the methods. This technique can be applied to puffy eyelids, in which natural fold formation is difficult using the non-incision method, and has the advantage of making a long-lasting fold. Furthermore, compared to the full incision, relatively simple procedures are performed through a much smaller incision, resulting in less postoperative edema and reduced downtime.

In addition to this, the authors published a technique combining the non-incision and partial incision techniques [12]. This method, involving 3 stab incisions and 1 small lateral incision, was used in those who requested the non-incision method or as a way to avoid the full-incision method in patients with puffy eyelids due to subcutaneous fat tissue, not thick skin. The orbital fat was removed through the small lateral incision and a suture was inserted through this site, passing through the dermis and tarsus sequentially via the other stab incisions for fixation. The continuous buried suture was performed by tying at the first lateral incision site. Using this method, the authors were able to perform an operation that enabled quick recovery and easy correction, even for patients with puffy eyelids, thereby incorporating the advantages of the non-incision method. Moreover, the incidence of cysts and granuloma was reduced because the suture knots could be buried more deeply than is possible in the non-incision method.

However, even with this method, a few cases of fold loosening were observed during the follow up 2-8 yrs, especially on the medial side. It is presumed that the reason for this is the lack of a levator musculoaponeurotic component on the medial side compared to the lateral side [13].

This method allows the orbital fat and orbicularis muscle to be removed more evenly and the medial fold to be formed more securely than is possible in the previously reported method [12], through a small incision on the medial side. Additionally, in this method, 2 small incisions are placed on both the medial and lateral limbus, reducing the possibility of levator injury. Simultaneously, fixation of the dermis and tarsus was performed at more points than previously to achieve a more secure adhesion, making it possible to reduce the possibility of fold loosening or loss.

The authors report an extensive case series with a review of the literature, because this technique, which was a modification and further development of existing surgical techniques, is thought to have led to good results. 


\section{PATIENT CONSENT}

Patients provided written consent for the use of their images.

\section{REFERENCES}

1. Cho BC, Byun JS. New technique combined with suture and incision method for creating a more physiologically natural double-eyelid. Plast Reconstr Surg 2010;125:324-31.

2. Jinghe Z, Huifang $X$, Lihong $W$, et al. Three mini-incision double-eyelid blepharoplasty. Ann Plast Surg 2014;72:141-4.

3. Yang SY. Oriental double eyelid: a limited-incision technique. Ann Plast Surg 2001;46:364-8.

4. Fakhro A, Yim HW, Kim YK, et al. The evolution of looks and expectations of Asian eyelid and eye appearance. Semin Plast Surg 2015;29: 135-44.

5. Lam SM. Mikamo's double-eyelid blepharoplasty and the westernization of Japan. Arch Facial Plast Surg 2002;4:201-2.

6. Lam SM. Asian blepharoplasty. Facial Plast Surg Clin North Am 2014;
22:417-25.

7. Lee CK, Ahn ST, Kim N. Asian upper lid blepharoplasty surgery. Clin Plast Surg 2013;40:167-78.

8. Ma FY, Cheng MS. Mini-incision double eyelidplasty. Aesthet Surg J 2010;30:329-34.

9. Lam SM, Kim YK. Partial-incision technique for creation of the double eyelid. Aesthet Surg J 2003;23:170-6.

10. Chuangsuwanich A. Short incisional double-eyelid blepharoplasty for Asian patients. Aesthet Surg J 2006;26:280-6.

11. Zhang MY, Yang H, Ding SL, et al. Construction of a double eyelid: an uncut strip of orbicularis removed through three mini-incisions. Aesthetic Plast Surg 2013;37:22-8.

12. Yoon IM, Hong JK, Yoo G. Double eyelid operation using partial incision and continuous buried suture. J Korean Soc Plast Reconstr Surg 2003;30:674-6.

13. Kim YS, Hwang K. Size and location of the superior transverse ligament and muscle-aponeurosis junction of the levator palpebrae superioris. J Craniofac Surg 2016;27:1098-100. 